\title{
Image Contour Extraction Method based on Computer Technology
}

\author{
Li Huanliang
}

Linyi University, Linyi, Shandong, 276400

Keywords: Computer technology; Image contour; Extraction method

\begin{abstract}
Effectively apply computer technology in image contour extraction can not only automatically detect surface image defects, but can better process image contour and realize the function of extracting single pixel image edge profile with high precision. It can meet the measurement requirements based on computer vision and play an active application value. This article is to discuss image contour extraction method with computer technology.

In computer vision measurement, image contour extraction is the key to its measurement. Computer technology can be used to extract the contour information in the image, which not only can automatically sort images' gray level, but can automatically detect surface defects in images. Better extract processing of images and improve image value. This paper makes in-depth internal image contour extraction and computer technology problem analysis, and analyzes the effective application of computer technology in image contour extraction.
\end{abstract}

\section{COMPUTER TECHNOLOGY APPLICATION VALUE IN IMAGE CONTOUR EXTRACTION}

With the improving of current computer information technology, we can use tools to get digital image information in computer outstanding processing field, and at the same time take a variety of image processing technologies for image contour information and extract them [1]. The outline of the image is a basic feature of the image, carrying most of the information of the image, and is often applied to the higher level image application. Based on computer technology, image contour separation not only can obtain image feature parameters in image processing, and can analyze and adjust image gray level through a variety of operators (Sobel operator, Laplace operator, Canny operator), thus realize the image edge contour extraction. It has practical application feasibility [2]. Image contour extraction based on computer technology is based on content image analysis. Use computer technology to extract the typical characteristics of the image, such as using important line, contour in the high threshold detecting image. Extraction system's line and contour are the several elements which can reflect the most image characteristics [3]. Use low threshold to ensure the easily lost details in image contour to make sure richer extract image edge and improve image contour extraction effect.

\section{THE APPLICATION DEMAND OF COMPUTER TECHNOLOGY IN IMAGE CONTOUR EXTRACTION}

Use computer for image processing. Its purpose is to get more suitable images for people to observe and recognize or automatically recognize and understand images by computer. Use computer technology, according to LOG operator, realize image frequency domain optimization, solve the contradiction between it and airspace optimization, and simplify the calculation method of computer image contour extraction. At the same time, in image extraction zero examination, according to the isotropic characteristic, guarantee the verge closure in image extraction process. The extracted image better conforms to human eye visual effect. Use computer analysis of the basic image features and attributes so that you can draw images and get better image contour extraction effect. 


\section{THE APPLICATION STRUCTURE DESIGN OF COMPUTER TECHNOLOGY IN IMAGE CONTOUR EXTRACTION}

Image contour often carries most of the information. The image outline exists in image irregular structure and unstable pixels. Of course, some exist in signal mutations. Apply computer technology to realize image contour extraction, and its overall design flow chart is shown in Figure 1 as follows:
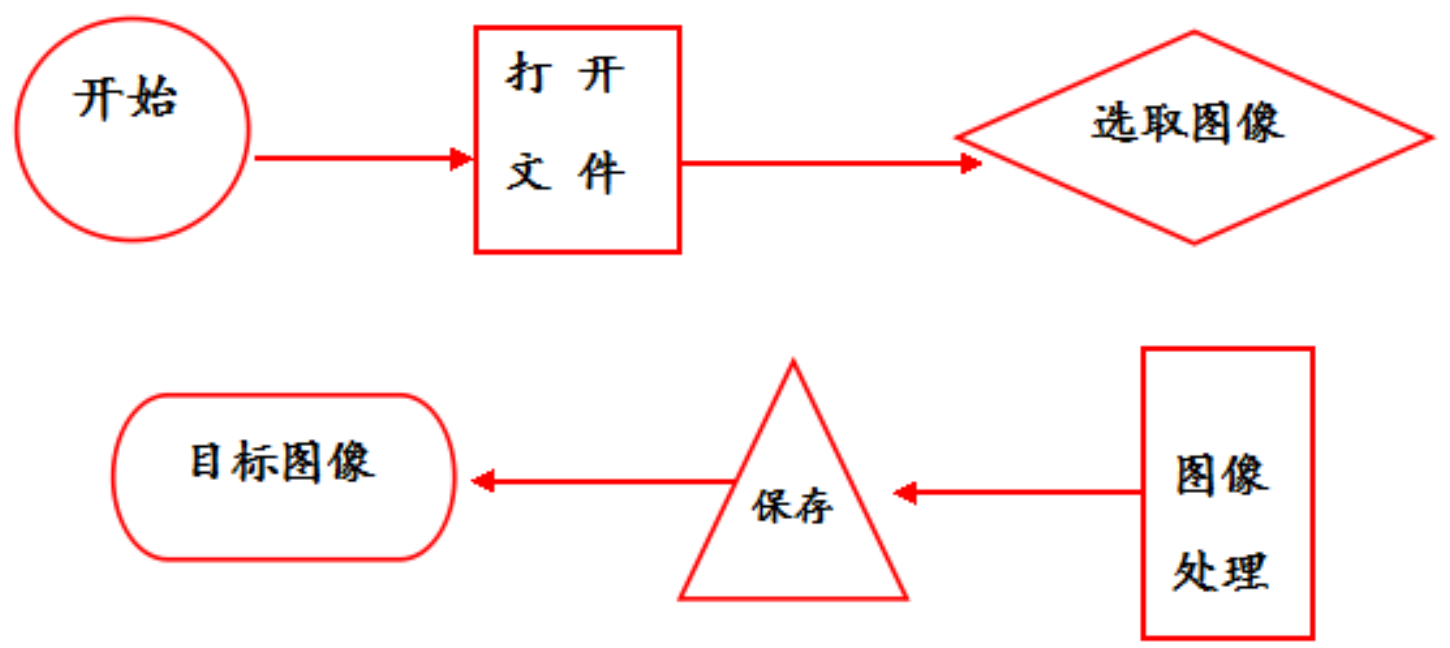

Figure 1 System flow chart

Apply computer QT5. 0+vs2012+0penCV3. 0 framework to help compatible OpenCV image processing and rendering [4]. This system is mainly composed of MyClass class and MyClass.ui class. QMainWindow in QT provides a menu bar such as toolbar and the main application window of the status bar. In MyClass class, debug and modify each function to realize the extraction of the image. In the overall design of the system, ensure that the design style is consistent. The structure should be clear and reasonable. Make modular management, and the interface should be clear to make users understand at a glance, and easy for user action. At the same time, make the developed code concise, easy to manage, easy to modify and easy for the expansion [5]. And we must have perfect data input and output with good fault tolerance. It won't collapse because of user's operation procedure. Every step operation is prompt, simple and clear, and it can remind the user to rectify.

\section{THE APPLICATION ANALYSIS OF COMPUTER TECHNOLOGY IN IMAGE CONTOUR EXTRACTION}

\section{A. Contour extraction design}

In image contour extraction, adopt computer technology. Get effective contour extraction information. In the following picture 2, for example, use computer to extract image contour.

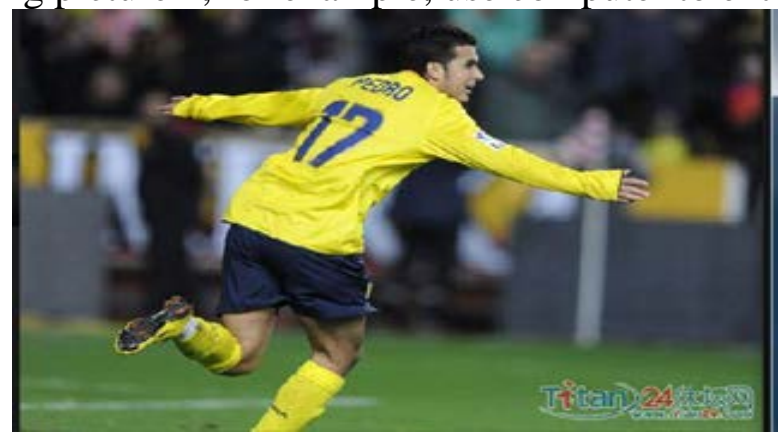

Figure 2 Extract contour images

Based on computer technology, image contour extraction can define different image contour according to the performance of different image contour method [6]. For computer technology, the use of OpenCV sequence to store contour information. Each element in the sequence is locations 
reflect of image curve [7]. As long as simply imagine contour as a series of points using CvSeq, that is ok. In image contour extraction, use computer technology to extract image contour. It is shown in Figure 3 below.

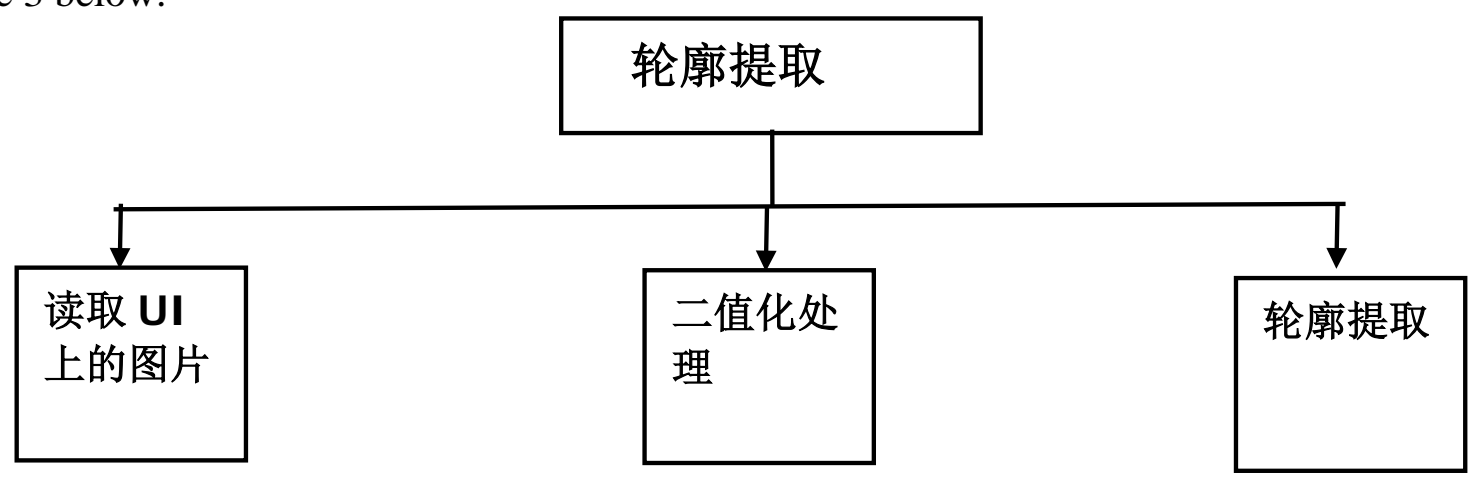

Figure 3 Contour extraction function diagram

\section{B. Specific extraction method}

For image contour extraction, we can use OpenCV software based on computer technology, use FindContours () function contour extraction [8]. The following Figure 3 is the resolution rendering after contour extraction. In OpenCV, express the outline with outline tree, thus encode inclusion relation into the tree structure.

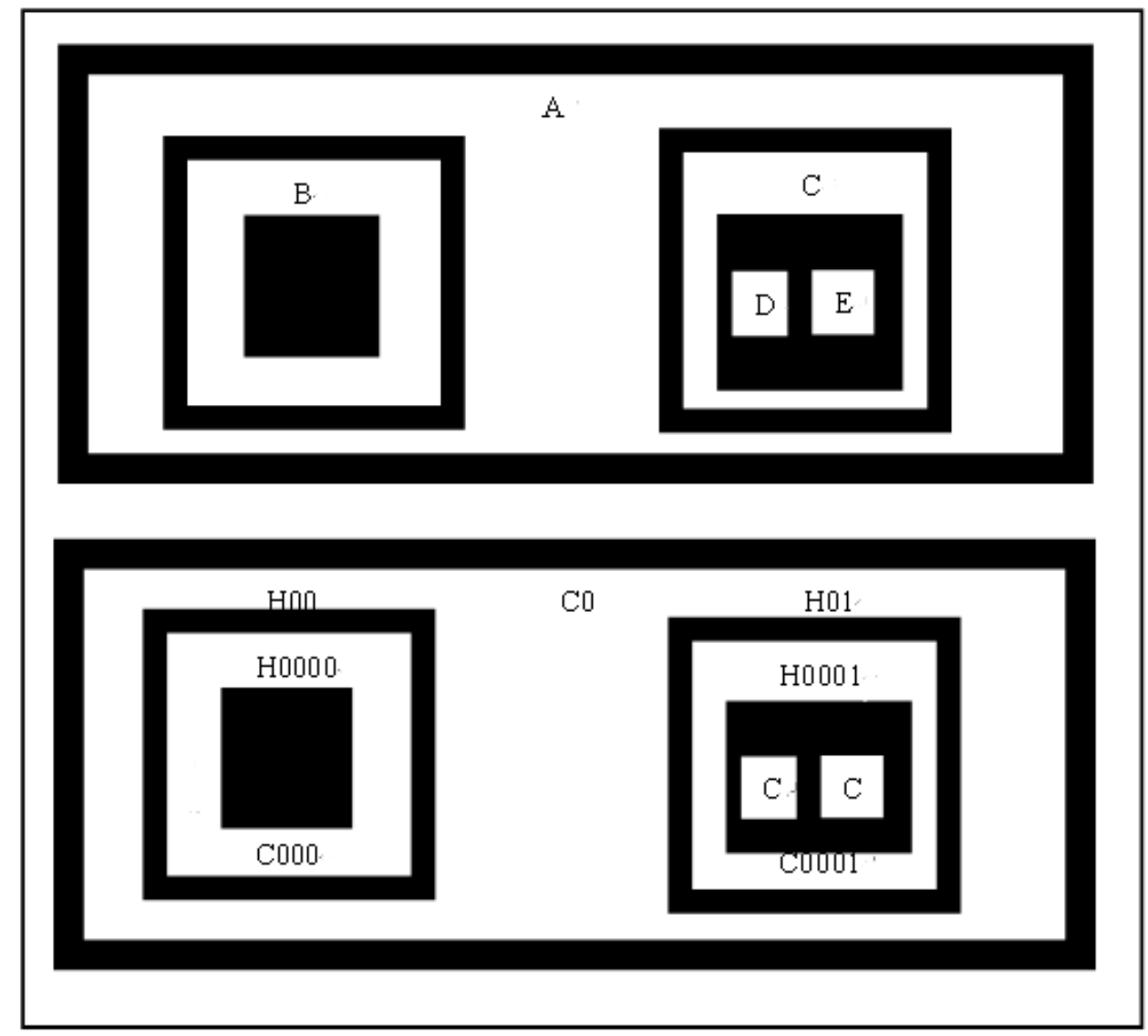

Figure 4 Contour image resolutions

We can see that set $\mathrm{C}$ in the picture as the inner contour, $\mathrm{H}$ as the outer contour. Based on two contour extractions, in different operator, their implementation method can represent respectively the outer boundary and outline border. In this figure contour tree, the outline of a root node is called c0, and the holes h00 and h01-2 are its word child nodes. These outlines directly include outlines, called their child nodes; Different ways of outline tree represent different outline storage ways.

\section{Code implementation}

From the binary image, based on FindContours () image contour extraction function, get edge pixels image, explain the needed outline type and the hoped return value form. According to canny 
detection operator parameters, image contour extraction results are obtained. The following is the relative code for the implementation of image contour extraction based on computer technology:

Void MyClass: : FindContours ()

\{

If (! Image. The empty ()) / / judge whether there is a picture

\{

RNG rng (12345);

int thresh = 100; / / contour extraction

int max_thresh $=255$; / / a maximum contour extraction

/ / the original conversion

Mat src. Mat src_gray;

src = image; / / picture link

cvtColor (src, src_gray, CV_BGR2GRAY); / / binary

blur (src_gray src_gray, Size (3, 3)); / / wipe out dryness

/ / / create the form

namedWindow ("contour extraction", WINDOW_AUTOSIZE);

imshow ("contour extraction", src);

Mat canny_output; / / initialize dst

/ / define points and vector

vector $<$ vector $<$ Point $>>$ contours.

vector $<$ Vec4i $>$ hierarchy;

Canny (src_gray canny_output, thresh, thresh * 2, 3); / / use Canny operator detect edge, also use other edge operator of course

findContours (canny_output, contours, hierarchy, RETR_TREE CHAIN_APPROX_SIMPLE, Point $(0,0))$; / / profile find

/ / / draw the contour

Mat drawing = Mat: : zeros (canny_output. The size (), CV_8UC3);

/ / iterate through all the top outlines, randomly generate color value part drawing for each connection

for (int I = 0; $\mathrm{I}<$ contours. The size ()$; \mathrm{i}++)$

\{

Scalar color $=$ Scalar $($ rng.uniform $(0,255)$, rng.uniform $(0,255)$, rng.uniform(0,255) );

drawContours ( drawing,contours, i, color, 2, 8, hierarchy, 0, Point () ) ; \}

imshow ("contour extraction", drawing); / / display the results in the form

\}

else

\{

QMessageBox: : information (this, tr (" ERROR!"),

tr (" both please click picture! "). Arg ("'")); / / pop-up extract box

\}

\}

Extract image edge, and get the following result, which is shown in Figure 4: 


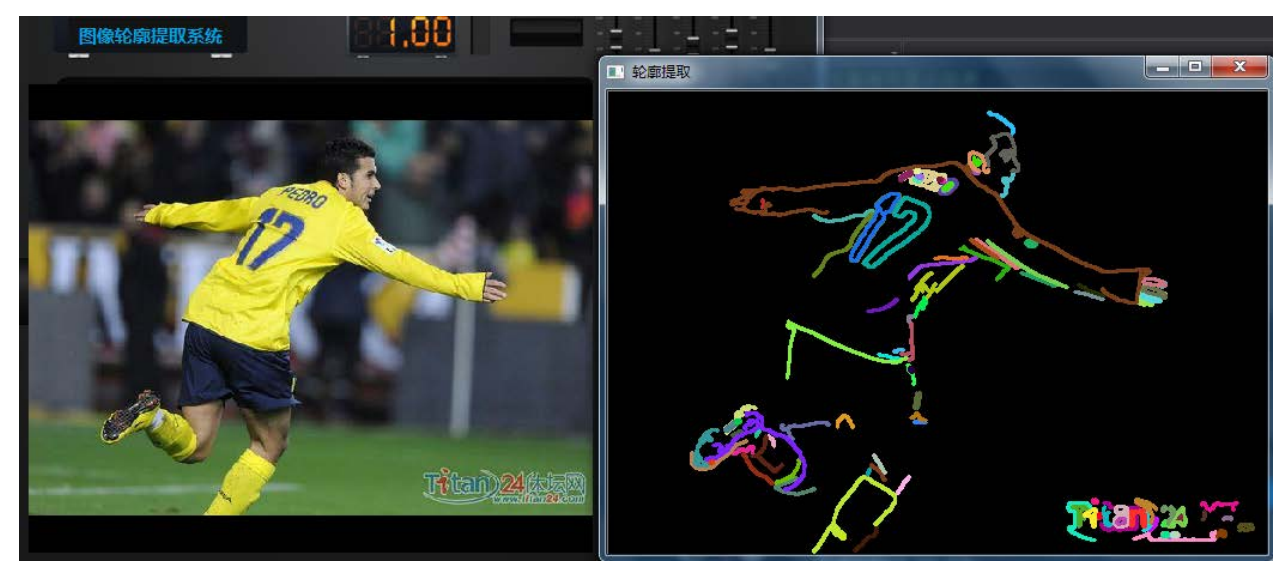

Figure 4 Contour extraction effects

According to canny operator parameters, the image contour demanded can be got after extracting. In image edge extraction, use computer technology and image contour extraction accuracy can reach $98.0 \%$. It effectively meets the actual needs of image engineering surveying and plays an active application value.

\section{Conclusion}

To sum up, in view of the problems existing in traditional image edge detection, conduct studies under the environment of VC. Combined with computer technology, use gray threshold method for image segmentation, and binary image defect was repaired with mathematical morphology method. Optimize design contour extraction method in order to improve image processing effect. Through the study, it is found that after computer technology is applied to image contour extraction, the accuracy is as high as $98.0 \%$, which can meet the practical need of engineering survey. So it can be seen in image contour extraction that application of computer technology in image contour extraction helps to achieve the best image processing effect, improves the image value, and plays a positive effect.

\section{REFERENCE}

[1] Yuan Da, Zhang Caiming, Li Jinjiang etc. High precision MR image contour extraction algorithm based on Mumford Shah model [J]. Journal of Computers, 2009, 32 (2) : 268-274.

[2] Yang Dan, You Lei, Zhang Xiaohong, etc. The fisheye image contour extraction algorithm based on region growing [J]. Computer Engineering, 2010, 4 (8) : 217-218221.

[3] Li Jinjuan. Image contour extraction scheme research and implementation based on iterative technology [J]. Science, Technology and Engineering, 2013, 13 (28) : 8325-8328,8338.

[4] Sun Jun, Li Chunlei, Liu Zhoufeng, etc. SAR image contour extraction algorithm based on wavelet transform and GVF model [J]. Journal of Henan University (Natural Science Edition), 2008, 38 (4) : 414-417.

[5] Han Huishan, Zhao Chunyuan, Zhao Jiawei etc. The monitoring image contour extraction algorithm based on dynamic mathematical morphology [C] / / China's Institutions of Higher Learning Power System and Its Automation Major 27th Session of Academic Essays. 2011:1-5.

[6] Wang Yanxia. Key techniques of image contour extraction and three-dimensional reconstruction [D]. Chongqing University, 2010.

[7] Chen Zhikun, Pan Xiaodi, Wang Fubin etc. Image contour extraction method researches based on Radon transform [J]. Journal of Sensors and Micro Systems, 2010, 29 (2) : 63-65 of.

[8] Wu Songcheng. Image contour extraction parallel implementation based on CUDA [J]. Modern Computer: Second Edition, 2012, (6) : 55-57, 21. 